Internat. J. Math. \& Math. Sci.

Vol. 24, No. 1 (2000) 11-27

S0161171200002751

(C) Hindawi Publishing Corp.

\title{
THE KOSTANT PARTITION FUNCTIONS FOR TWISTED KAC-MOODY ALGEBRAS
}

\author{
RANABIR CHAKRABARTI and THALANAYAR S. SANTHANAM
}

(Received 2 March 1998)

\begin{abstract}
Employing the method of generating functions and making use of some infinite product identities like Euler, Jacobi's triple product and pentagon identities we derive recursion relations for Kostant's partition functions for the twisted Kac-Moody algebras.
\end{abstract}

Keywords and phrases. Partition function, Kac-Moody algebra, generating functions.

2000 Mathematics Subject Classification. Primary 22E60, 11P83.

1. Introduction. The weight multiplicity $M_{\Lambda}(m)$ of the weight $m$ belonging to the irreducible representation with the highest weight $\Lambda$ of the affine Lie algebra can be computed using the Kostant-Kac formula (Kac [3])

$$
M_{\Lambda}(m)=\sum_{s \in W} \delta_{s} K[s(\Lambda+\rho)-(m+\rho)],
$$

where $W$ stands for the Weyl group for the affine Lie Algebras and $\rho$ (analog of half the sum of positive roots of the finite dimensional Lie algebra) is defined by

$$
\left(\rho, a_{i}\right)=\frac{1}{2}\left(a_{i}, a_{i}\right), \quad i=0,1,2, \ldots, \ell,
$$

$a_{i}$ 's are the simple roots and $\delta_{S}= \pm 1$ depending on the nature of the permutation resulting from the action of $W$. The Kostant partition function (KPF) $K(\Omega)$ is defined as the number of ways $\Omega$, which is given by a fixed linear integral combination of the simple roots,

$$
\Omega=\sum_{i=0}^{\ell} n_{i} a_{i}, \quad n_{i} \in \mathbb{Z}_{+},
$$

can be partitioned into non-negative linear integral combination of the positive roots. For the affine algebras, these positive roots are infinite in number.

Kostant's partition function $K\left(n_{0}, n_{1}, \ldots, n_{\ell}\right)$ appears as the expansion coefficient in the Taylor expansion of the generating function

$$
G\left(x_{0}, x_{1}, \ldots, x_{\ell}\right)=\sum_{\left\{n_{i}\right\}} K\left(n_{0}, \ldots, n_{\ell}\right) x_{0}^{n_{0}} x_{1} n_{1} \cdots x_{\ell} n_{\ell}, \quad\left|x_{i}\right|<1 \text { for all } i .
$$

The generating function $G$ which can be constructed from the knowledge of the simple and positive roots of the affine algebra taking proper account of their multiplicities may be easily shown to be equal to $\Delta^{-1}$, where $\Delta$ is the denominator function in the character formula [4]. 
In [5] we developed a method using the generating functions to obtain the KPF for the affine Lie algebras. The method consists in recognizing that the inverse of the generating function can be expressed as an infinite product identity. This results in recursion relations for the KPF. For simple cases like the Virasoro algebra and the affine algebra $A_{1}^{(1)}$, these relations are nothing but Euler's equation and Carlitz equations [1] for the partition functions.

Kac and Peterson [4] have obtained the KPF for the affine algebra $A_{1}^{(1)}, A_{1}^{(1)}$ and $A_{2}^{(2)}$ using the properties of the Weyl group.

It has been shown [5] that using the method of generating functions we can get the expression of Kac-Peterson for the KPF for $A_{1}^{(1)}$ if we make use of the number theoretic identity of Tannery and Molk [6] derived as a $\theta$-function identity. We had earlier found the generalization of the T. M. identity for the case of $A_{2}^{(1)}$.

In the present article we develop the method of the generating functional for all of the twisted affine algebras. The twisted affine algebras consist of the series $A_{2 \ell}^{(2)}(\ell \geq 1)$, $A_{2 \ell-1}^{(2)}(\ell \geq 3)$ and the special cases of $E_{6}^{(2)}$ and $D_{4}^{(3)}$. We construct the generating functional for all of the above cases and then derive the recursive relations for the KPF in some typical examples.

A twisted affine algebra $X_{N}^{(k)}$ admits an automorphism $\tau$ of the Dynkin diagram of the Lie algebra $X_{N}$ of the order $k$ so that

$$
\tau^{k}=1
$$

From the Lie algebra $X_{N}$, an invariant eigenspace $g^{(0)}$ on which $\tau=1$ may be projected out. A Kac-Moody extension of $g^{(0)}$ is possible by the addition of an extra simple root $a_{0}$ whose choice can be made by following the prescription of Goddard and Olive [2]. For the series $A_{2 \ell}^{(2)}$ the extra root is

$$
a_{0}=\left(-\phi, 0, \frac{1}{2}\right)
$$

where $\phi$ is the highest short root of $A_{2 \ell}$. This is twice of the highest short root of the corresponding invariant subalgebra $B_{\ell}$. For other twisted affine algebras, $a_{0}$ is given by

$$
a_{0}=\left(-\phi, 0, \frac{1}{2}\right)
$$

where $\phi$ is the highest short root of the corresponding invariant subalgebra $g^{(0)}$.

Several infinite product identities, which we frequently use are listed below:

(I) Euler's identity

$$
\phi(q) \equiv \prod_{n \geq 1}\left(1-q^{n}\right)=\sum_{k \in \mathbb{Z}}(-1)^{k} q^{(k / 2)(3 k+1)},
$$

(II) Jacobi's triple product identity

$$
\begin{aligned}
T(u, v) & \equiv \prod_{n \geq 1}\left(1-u^{n} v^{n}\right)\left(1-u^{n} v^{n-1}\right)\left(1-u^{n-1} v^{n}\right) \\
& =\sum_{k \in \mathbb{Z}}(-1)^{k} u^{(k / 2)(k+1)} v^{(k / 2)(k-1)},
\end{aligned}
$$


(III) the quintuple product identity

$$
\begin{aligned}
Q(u, v) \equiv & \prod_{n \geq 1}\left(1-u^{2 n} v^{n}\right)\left(1-u^{2 n-1} v^{n-1}\right)\left(1-u^{2 n-1} v^{n}\right) \\
& \times\left(1-u^{4 n-4} v^{2 n-1}\right)\left(1-u^{4 n} v^{2 n-1}\right) \\
= & \sum_{k \in \mathbb{Z}}\left[u^{\left(3 k^{2}-2 k\right)} v^{\left(3 k^{2}+k\right) / 2}-u^{\left(3 k^{2}-4 k+1\right)} v^{\left(3 k^{2}-k\right) / 2}\right] .
\end{aligned}
$$

The plan of the paper is as follows. In Section 2, we review our earlier work on the generating functional approach to the KPF for the algebra $A_{2}^{(2)}$. The equivalence of our work with the results of Kac and Peterson [4] dictates an infinite product identity for each affine algebra. Here we derive the identity for $A_{2}^{(2)}$. To our knowledge this identity has not appeared earlier. The significance of these identities is under investigation. Sections 3, 4, 5, and 6 contain our discussions for the KPF for the $A_{2 \ell}^{(2)}(\ell \geq 2), A_{2 \ell-1}^{(2)}(\ell \geq 3), D_{\ell+1}^{(2)}(\ell \geq 2)$ and the special cases of $E_{6}^{(2)}$ and $D_{4}^{(3)}$, respectively. Finally we conclude in Section 7 .

2. Partition function for $A_{2}^{(2)}$. The Cartan matrix for this case $A_{2}^{(2)}$ is

$$
\left(\begin{array}{cc}
2 & -4 \\
-1 & 2
\end{array}\right)
$$

With the set of the positive roots given by

$$
\Delta_{+}=\left\{\begin{array}{l}
4 n a_{0}+(2 n-1) a_{1} \\
4(n-1) a_{0}+(2 n-1) a_{1} \\
(2 n-1) a_{0}+n a_{1} \\
(2 n-1) a_{0}+(n-1) a_{1} \\
2 n a_{0}+n a_{1},
\end{array}\right.
$$

where $a_{0}$ and $a_{1}$ are the simple roots of $A_{2}^{(2)}$ and $n=\mathbb{Z}_{+}-\{0\}$. The Diophantine equations yielding the Kostant partition function $K\left(n_{0}, n_{1}\right)$ are

$$
\begin{aligned}
& n_{0}=\sum_{n}\left[4 n p_{n}+4(n-1) q_{n}+(2 n-1) r_{n}+(2 n-1) \delta_{n}+2 n t_{n}\right], \\
& n_{1}=\sum_{n}\left[(2 n-1) p_{n}+(2 n-1) q_{n}+n r_{n}+(n-1) \delta_{n}+n t_{n}\right],
\end{aligned}
$$

where $p_{n}, q_{n}, \ldots, t_{n}$ are non-negative integral variables. The generating functional for the partitions is

$$
G\left(x_{0}, x_{1}\right)=\left[Q\left(x_{0}, x_{1}\right)\right]^{-1}=\sum_{n_{0}, n_{1}=0}^{\infty} K_{A_{2}^{(2)}}\left(n_{0}, n_{1}\right) x_{0}^{n_{0}} x_{1}^{n_{1}} .
$$

The second equality is valid when $\left|x_{0}\right|<1$ and $\left|x_{1}\right|<1$. Using the quintuple product identity we can derive the recursive relation

$$
\begin{aligned}
& \sum_{m \in \mathbb{Z}} K_{A^{(2)}}\left(n_{0}-3 m^{2}+2 m, n_{1}-\frac{3 m^{2}+m}{2}\right) \\
& \quad-K_{A_{2}^{(2)}}\left(n_{0}-3 m^{3}+4 m-1, n_{1}-\frac{3 m^{2}-m}{2}\right)=\delta_{n_{0}, 0} \delta_{n_{1}, 0} .
\end{aligned}
$$


In an alternate approach Kac and Peterson [4] derived the explicit formula

$$
\begin{aligned}
K_{A^{(2)}}\left(n_{0}, n_{1}\right)= & \sum_{k \geq 1} p^{(2123)}\left[(2 k-1) n_{0}-4(k-1) n_{1}-(k-1)(3 k-1)\right] \\
& -\sum_{k \geq 1} p^{(53)}\left[k n_{0}-(2 k-1) n_{1}-\frac{1}{2} k(3 k-1)\right] .
\end{aligned}
$$

Using (2.4) and the expression for $K_{A_{2}^{(2)}}$ in (2.6), now we derive an identity for the inverse of the quintuple product structure

$$
\begin{aligned}
{\left[Q\left(u, w^{2}\right)\right]^{-1}=} & {\left[\phi(u w)^{-2123} \sum_{k \geq 0}(u w)^{k(3 k+2)} \frac{u^{2 k} w^{2 k+1}}{1-u^{2 k} w^{2 k+1}}\right.} \\
& \left.-\phi\left(u^{2} w^{2}\right)^{-53} \sum_{k \geq 1}(u w)^{k(3 k-1)} \frac{u^{2 k-1} w^{2 k}}{1-u^{2 k-1} w^{2 k}}\right]
\end{aligned}
$$

or

$$
\begin{aligned}
{\left[Q\left(u, w^{2}\right)\right]^{-1}=} & {\left[\phi(u w)^{-2123} \sum_{k \geq 0}(u w)^{k(3 k+2)} \frac{1}{1-u^{-2 k} w^{-(2 k+1)}}\right.} \\
& \left.-\phi\left(u^{2} w^{2}\right)^{-53} \sum_{k \geq 1}(u w)^{k(3 k-1)} \frac{1}{1-u^{-2 k-1} w^{-2 k}}\right] .
\end{aligned}
$$

The right-hand side of the identity (2.8) means the following. If we compare the expansion coefficients of the terms $u^{n_{0}} w^{2 n_{1}}$ for $n_{0}<2 n_{1}$ the first expression in the right-hand side gives identical results as this left-hand side. The domain of convergence in this case is $u, w<1$, when the expansion coefficients of the terms $u^{n_{0}} w^{2 n_{1}}$ for $n_{0} \geq 2 n_{1}$ are to be compared, we choose the second expression in the right-hand side. This has a domain of convergence $u, w>1$.

3. Partition functions for $A_{2 \ell}^{(2)}$. The example of the algebras $A_{2 \ell}^{(2)}$ is distinct from the other cases as in the former generating functionals for KPF get contributions for each of the roots of the Lie algebra $A_{2 \ell}$ invariant under the automorphism of the Dynkin diagram-equal to a factor that is inverse of the quintuple product structure. This can be directly traced to the fact that no simple root of $A_{2 \ell}$ remain invariant under the outer automorphism. As a consequence the real roots of $A_{2}^{(2)}$ have their different sizes with the square of the roots having the ratio $4: 2: 1$. The largest roots of the affine algebra always contain the smallest simple root in a multiple of 4 . This forces the appearance of the inverse of the quintuple product structure for the generating functional.

The Lie algebra has the simple roots $\alpha_{1}, \ldots, \alpha_{2}$ and admits an automorphism of its Dynkin diagram

$$
\alpha_{i} \leftrightharpoons \alpha_{2 \ell-i+1},
$$

where $i=1,2, \ldots, \ell$. The $\ell$ positive roots of $A_{2 \ell}$ invariant under this automorphism are

$$
\alpha_{1}+\alpha_{\ell+1}, \alpha_{\ell-1}+\alpha_{\ell}+\alpha_{\ell+1}+\alpha_{\ell+2}, \ldots, \alpha_{1}+\cdots+\alpha_{2 \ell}
$$


The subalgebra of $A_{2 \ell}$, invariant under the other automorphism (3.1) is $B_{\ell}$ and its simple roots $\beta_{1}, \ldots, \beta_{\ell}$ may be projected as

$$
\beta_{i}=\frac{1}{2}\left(\alpha_{i}+\alpha_{2 \ell-i+2}\right),
$$

where $i=1, \ldots, \ell$. For later use we identify the positive roots of $B_{\ell}$. The short roots are

$$
\stackrel{\circ}{\Delta}_{S}=\left\{\beta_{\ell}, \beta_{\ell-1}+\beta_{\ell}, \ldots, \beta_{1}+\beta_{2}+\cdots+\beta_{\ell}\right\} .
$$

Each of the invariant roots of $A_{2 \ell}$ in (3.2) correspond to a short root of $\beta_{i}$. We divide, $\Delta$, the long roots of $\beta_{\ell}$ into two groups.

(I) The roots of the subalgebra $A_{\ell-1}\left(c_{\ell}\right),(\ell(\ell-1) / 2)$ in number are

$$
\beta_{1}, \ldots, \beta_{\ell-1}, \beta_{1}+\beta_{2}, \ldots, \beta_{\ell-2}+\beta_{\ell-1}, \ldots, \beta_{1}+\beta_{2}+\cdots+\beta_{\ell-1} .
$$

(II) The rest of the long roots of $B_{\ell}$, consisting of several series are:

$$
\begin{gathered}
\beta_{\ell-1}+2 \beta_{\ell}, \beta_{\ell-2}+\beta_{\ell-1}+2 \beta_{\ell}, \ldots, \beta_{1}+\beta_{2}+\cdots+2 \beta_{\ell}, \\
\beta_{\ell-2}+2 \beta_{\ell-1}+2 \beta_{\ell}, \beta_{\ell-3}+\beta_{\ell-2}+2 \beta_{\ell-1}+2 \beta_{\ell}, \ldots, \\
\beta_{1}+\cdots+\beta_{\ell-2}+2 \beta_{\ell-1}+2 \beta_{\ell}, \\
\beta_{1}+2 \beta_{2}+\cdots+2 \beta_{\ell} .
\end{gathered}
$$

The $(\ell+1)$ simple roots of the algebra $A_{2 \ell}^{(2)}$ may be directly written as

$$
a_{0}=\left(-2 \beta_{1}-2 \beta_{2}-\cdots-2 \beta_{\ell}, 0, \frac{1}{2}\right), \quad a_{1}=\left(\beta_{1}, 0,0\right), \quad a_{\ell}=\left(\beta_{\ell}, 0,0\right) .
$$

The Cartan matrix for the algebra $A_{2 \ell}^{(2)}$,

$$
\left(\begin{array}{cccccccc}
2 & -2 & 0 & 0 & & & & \\
-1 & 2 & -1 & 0 & & & 0 & \\
0 & -1 & 2 & -1 & & & \\
& & & & \ddots & & \\
& & & & & & 2 & -2 \\
& & 0 & & & & & \\
& & & & & & -1 & 2
\end{array}\right)
$$

admits a null vector $(1,2, \ldots, 2)$ which reflects the structure of the imaginary root

$$
\delta=\left(0,0, \frac{1}{2}\right)=a_{0}+2 a_{1}+\cdots+2 a_{\ell} .
$$

The complete set of the imaginary roots of $A_{2 \ell}^{(2)}$ are given by $n \delta$, where $n=\mathbb{Z}_{+}-\{0\}$ and each imaginary root has a degeneracy $\ell$. The positive real roots of $A_{2 \ell}^{(2)}$ have two origins. The first set which has its genesis in the roots of $A_{2 \ell}$ listed in (3.2), which are invariant under the outer automorphism, is given by

$$
\left( \pm 2 \beta_{\ell}, 0, n\right),\left( \pm 2 \beta_{\ell-1} \pm 2 \beta_{\ell}, 0, n\right), \ldots,\left( \pm 2 \beta_{1} \pm 2 \beta_{2} \pm \cdots \pm 2 \beta_{\ell}, 0, n\right),
$$

where $n=\mathbb{Z}_{+}+(1 / 2)$. The outer set of positive roots are of the type 


$$
(\beta, 0,0) \cup\left( \pm \beta, 0, \frac{n}{2}\right),
$$

where $n=\mathbb{Z}_{+}-\{0\}$ and $\beta$ is any positive root of $B_{\ell}$, belonging to the set (3.4), (3.5), and (3.6). When $\beta \in \Delta_{S}$, the length of the roots in (3.11) becomes $1 / 4$ of the length of the roots in (3.10).

Each of the sequences of the roots of $A_{2 \ell}$ contributes a multiplication factor to the generating function $G_{A_{2 \ell}^{(2)}}\left(x_{0}, x_{1}, \ldots, x_{\ell}\right)$. Let us consider the following root sequence: $\left( \pm 2 \beta_{i}, 0, n\right)$, where $n=\mathbb{Z}_{+}+(1 / 2) ;\left(\beta_{\ell}, 0,0\right) \cup\left( \pm \beta_{\ell}, 0, n / 2\right)$, where $n=\mathbb{Z}_{+}-\{0\}$ and the imaginary roots $(0,0, n / 2)$, where $n=\mathbb{Z}_{+}-\{0\}$ the last set is being considered with unit multiplicity. The contribution of the above sets are respectively

$$
\begin{gathered}
\prod_{n=1}^{\infty}\left[1-x_{\ell}^{4 n-4}\left(x_{0} x_{1}^{2} \cdots x_{\ell-1}^{2}\right)^{2 n-1}\right]^{-1}\left[1-x_{\ell}^{4 n}\left(x_{0} x_{1}^{2} \cdots x_{\ell-1}^{2}\right)^{2 n-1}\right]^{-1}, \\
\prod_{n=1}^{\infty}\left[1-x_{\ell}^{2 n-1}\left(x_{0} x_{1}^{2} \cdots x_{\ell-1}^{2}\right)^{n-1}\right]^{-1}\left[1-x_{\ell}^{2 n-1}\left(x_{0} x_{1}^{2} \cdots x_{\ell-1}^{2}\right)^{n}\right]^{-1}, \\
\prod_{n=1}^{\infty}\left[1-x_{\ell}^{2 n}\left(x_{0} x_{1}^{2} \cdots x_{\ell-1}^{2}\right)^{n}\right]^{-1} .
\end{gathered}
$$

These factors form together the inverse of the term $\left[Q\left(x_{\ell} x_{0} x_{1}^{2} \cdots x_{\ell-1}^{2}\right)\right]^{-1}$ of the quintuple product. Each of the $\ell$ series of roots in (3.10) along with a corresponding series of the type $\left(\beta\left(\epsilon \stackrel{\circ}{S}_{S}\right), 0,0\right) \cup\left( \pm \beta\left(\epsilon \stackrel{\circ}{S}_{S}\right), 0, n\left(\epsilon\left(\mathbb{Z}_{+} / 2\right)-\{0\}\right)\right)$ and set the imaginary roots $\left(0,0, n\left(\epsilon\left(\mathbb{Z}_{+} / 2\right)-\{0\}\right)\right)$ considered with unit multiplicity, contributes a factor $Q^{-1}$ to the generating functional. This product grouped as

$$
\begin{aligned}
G_{s}\left(x_{0}, x_{1}, \ldots, x_{\ell}\right) & =\left[Q\left(x_{\ell}, y_{1}\right) Q\left(x_{\ell-1} x_{\ell}, y_{2}\right) \cdots Q\left(x_{2} \cdots x_{\ell}, y_{\ell-1}\right) Q\left(x_{1} \cdots x_{\ell}, x_{0}\right)\right]^{-1}, \\
y_{i} & =x_{0} x_{1}^{2} \cdots x_{\ell-i}^{2}, \quad i=1,2, \ldots, \ell-1,
\end{aligned}
$$

gives the full generating functional except for the contributions of the series related to the long roots of $\beta_{\ell}$.

The contribution to the generating functional due to the root of the type $(\beta, 0,0) \cup$ $( \pm \beta, 0, n / 2)$, where $n \in \mathbb{Z}_{+}$and $\beta \varepsilon \dot{\Delta}_{L}$ may be understood by considering a series say $\left(\beta_{1}, 0,0\right) \cup\left( \pm \beta_{\ell}, 0, n\left(\varepsilon\left(\mathbb{Z}_{+} / 2\right)-\{0\}\right)\right)$. It contributes to the generating functional the factor

$$
\prod_{n=1}^{\infty}\left[\left(1-q^{n} x_{1}\right)\left(1-q^{n} x_{1}^{-1}\right)\right]^{-1}\left(1-x_{1}\right)^{-1},
$$

where the variables $q$, related to the imaginary root is

$$
q=x_{0} x_{1}^{2} \cdots x_{\ell}^{2} .
$$

We rewrite (3.14) as

$$
\begin{aligned}
\prod_{n=1}^{\infty}\left(1-q^{n}\right) \prod_{n=1}^{\infty} & \left(\left[1-\left(x_{0} x_{1} x_{2}^{2} \cdots x_{\ell}^{2}\right)^{n} x_{1}^{n}\right]\left[1-\left(x_{0} x_{1} x_{2}^{2} \cdots x_{\ell}^{2}\right)^{n-1} x_{1}^{n}\right]\right. \\
& \left.\times\left[1-\left(x_{0} x_{1} x_{2}^{2} \cdots x_{\ell}^{2}\right)^{n} x_{1}^{n-1}\right]\right)^{-1}=\phi(q)\left[T\left(x_{1}, q x_{1}^{-1}\right)\right]^{-1} .
\end{aligned}
$$


Each of the $\left(\ell^{2}-\ell\right)$ long roots of $B_{\ell}$ causes such a multiplicative factor in the generating functional, which has the structure

$$
G_{L}\left(x_{0}, x_{1}, \ldots, x_{\ell}\right)=G_{A_{\ell-1}^{(1)}}\left(x_{0}, x_{1}, \ldots, x_{\ell-1} ; q\right) G_{L}^{\prime}\left(x_{0}, x_{1}, \ldots, x_{\ell}\right),
$$

where

$$
\begin{aligned}
G_{A_{\ell}^{(1)}}\left(x_{0}, x_{1}, \ldots, x_{\ell-1} ; q\right) & \\
=[\phi(q)]^{(\ell-1)(\ell-2) / 2} & {\left[T\left(x_{1}, q x_{1}^{-1}\right) \cdots T\left(x_{\ell-1}, q x_{\ell-1}^{-1}\right)\right.} \\
& \times T\left(x_{1} x_{2}, q x_{1}^{-1}, x_{2}^{-1}\right) \cdots T\left(x_{\ell-2} x_{\ell-1}, q x_{\ell-2}^{-1} x_{\ell-1}^{-1}\right) \\
& \left.\times T\left(x_{1} x_{2} \cdots x_{\ell}, q x_{1}^{-1} x_{2}^{-1} \cdots x_{\ell}^{-1}\right)\right]
\end{aligned}
$$

and

$$
\begin{aligned}
G_{L}^{\prime}\left(x_{0}, x_{1}, \ldots, x_{\ell}\right) & \\
=[\phi(q)]^{(\ell-1)(\ell-2) / 2}\{[ & T\left(x_{\ell-1}, x_{\ell}^{2}, q x_{\ell-1}^{-1} x_{\ell-2}^{-2}\right) \\
& \cdots T\left(x_{1} \cdots x_{\ell-1} x_{\ell}^{2}, q x_{1}^{-1} \cdots x_{\ell-2}^{-1} x_{\ell-1}^{-1} x_{\ell}^{-2}\right) \\
& \left.\left.\times T\left(x_{1} x_{2}^{2} \cdots x_{\ell}^{2}, q x_{1}^{-1} x_{2}^{-2} \cdots x_{\ell}^{-2}\right)\right]\right\}^{-1} .
\end{aligned}
$$

The contribution (3.18) has the same form as that of the generating functional for the KPF for $A_{\ell-1}^{(1)}$, which appears as a consequence of the roots of $A_{\ell-1}\left(\subset B_{\ell}\right)$ listed in (3.5). The full generating functional for an arbitrary algebra $A_{2 \ell}^{(2)}$ may be obtained as a product

$$
G_{A_{2 \ell}^{(2)}}=G_{S} G_{L} .
$$

The technique of obtaining the recursive relations for the KPF is the following. We first substitute the full generating functional (3.20) in (1.4) and multiply both sides with the Euler product, the triple and quintuple product with the appropriate argument. Then matching terms with a particular set of exponents for the $x$-variables, we obtain the recursive relations for the KPF. The result for $A_{4}^{(2)}$ reads as

$$
\begin{aligned}
\sum_{m, n, p, q \in \mathbb{Z}}(-1)^{m+n}[ & K\left(M-N_{1}, N-N_{1}, P-P_{1}\right)-K\left(M-M_{2}, N-N_{2}, P-P_{2}\right) \\
& \left.-K\left(M-M_{3}, N-N_{3}, P-P_{3}\right)+K\left(M-M_{4}, N-N_{4}, P-P_{4}\right)\right] \\
= & \sum_{k, \ell \in \mathbb{Z}}(-1)^{k+\ell} \delta_{M, E} \delta_{N, 2 E} \delta_{P, 2 E},
\end{aligned}
$$

where

$$
\begin{array}{ll}
M_{1}=L+\frac{3 p^{2}+p}{2}+\left(3 q^{2}+q\right)_{2}, & M_{2}=L+\frac{3 p^{2}-p}{2}+\frac{3 q^{2}+q}{2}, \\
M_{3}=L+\frac{3 p^{2}+p}{2}+\frac{3 q^{2}-q}{2}, & M_{4}=L+\frac{3 p^{2}-p}{2}+\frac{3 q^{2}-q}{2}, \\
N_{1}=R+3 p^{2}+p+3 q^{2}-2 q, & N_{2}=R+3 p^{2}-p+3 q^{2}-2 q, \\
N_{3}=R+3 p^{2}+p+3 q^{2}-4 q+1, & N_{4}=R+3 p^{2}-p+3 q^{2}-4 q+1, \\
P_{1}=S+3 p^{2}-2 p+3 q^{2}-2 q, & P_{2}=S+3 p^{2}-4 p+1+3 q^{2}-2 q, \\
P_{3}=S+3 p^{2}-2 p+3 q^{2}-4 q+1, & P_{4}=S+3 p^{2}-4 p+3 q^{2}-4 q+2,
\end{array}
$$


with

$$
\begin{gathered}
L=\frac{m}{2}(m+1)+\frac{n}{2}(n-1), \quad R=m^{2}+n^{2}, \quad S=m(m+1)+n(n+1), \\
E=\frac{k}{2}(3 k+1)+\frac{\ell}{2}(3 \ell+1) .
\end{gathered}
$$

The recursive formula for any other algebra may be obtained similarly.

4. Partition function for $A_{2 \ell-1}^{(2)}$. Unlike the case of $A_{2 \ell}^{(2)}$, the inverse of the quintuple product structure does not appear in the generating functional for the KPF for an $A_{2 \ell-1}^{(2)}, \ell \geq 3$ algebra. Instead, the inverse of the triple product and Euler product completely constitute the generating functional.

The Dynkin diagram of $A_{2 \ell-1}$, with the simple roots $\alpha_{1}, \alpha_{2}, \ldots, \alpha_{2 \ell-1}$ admits an automorphism defined by

$$
\alpha_{i} \leftrightharpoons \alpha_{2 \ell-i}
$$

where $i=1,2, \ldots,(\ell-1)$ and $\alpha_{\ell}$ is kept invariant. The $\ell$ roots invariant under this map are:

$$
\alpha_{\ell}, \alpha_{\ell-1}+\alpha_{\ell}+\alpha_{\ell+1}, \ldots, \alpha_{1}+\alpha_{2}+\cdots+\alpha_{2 \ell-1} .
$$

The subalgebra $C_{\ell}$ invariant under the map (4.1) may be constructed from the simple roots

$$
\begin{gathered}
\beta_{i}=\frac{\alpha_{i}+\alpha_{2 \ell-i}}{2}, \quad i=1,2, \ldots,(\ell-1), \\
\beta_{\ell}=\alpha_{\ell} .
\end{gathered}
$$

The positive roots of $C_{\ell}$ are listed below. The roots of $A_{2 \ell-1}$ invariant under the automorphism, as listed in (4.2) reappear as the long roots of $C_{\ell}$.

$$
\stackrel{\circ}{\Delta}_{L}=\left\{\beta_{\ell}, 2 \beta_{\ell-1}+\beta_{\ell}, 2 \beta_{\ell-2}+2 \beta_{\ell-1}+\beta_{\ell}, \ldots, 2 \beta_{1}+2 \beta_{2}+\cdots+2 \beta_{\ell-1}+\beta_{\ell}\right\} .
$$

For the purpose of easy identification we divide the set of short roots $\Delta_{S}$ of $C_{\ell}$ in several sequences. A subalgebra $A_{\ell-1}\left(\subset C_{\ell}\right)$ is composed of the simple roots $\beta_{1}, \beta_{2}, \ldots$, $\beta_{\ell-i}$. The positive roots for this $A_{\ell-1}$ are $\ell(\ell-1) / 2$ in number and are given by

$$
\beta_{1}, \ldots, \beta_{\ell-1}, \beta_{1}+\beta_{2}, \ldots, \beta_{\ell-2}+\beta_{\ell-1}, \ldots, \beta_{1}+\beta_{2}+\cdots+\beta_{\ell-1} .
$$

The rest of the short roots, also $\ell(\ell-1) / 2$ in number, are conveniently grouped as

$$
\begin{gathered}
\beta_{\ell-1}+\beta_{\ell}, \beta_{\ell-2}+\beta_{\ell-1}+\beta_{\ell}, \ldots, \beta_{1}+\cdots+\beta_{\ell-1}+\beta_{\ell}, \\
\beta_{\ell-2}+2 \beta_{\ell-1}+\beta_{2}, \beta_{\ell-3}+\beta_{\ell-2}+2 \beta_{\ell-1}+\beta_{\ell}, \ldots, \beta_{1}+\cdots+\beta_{\ell-2}+2 \beta_{\ell-1}+\beta_{\ell}, \\
\ldots, \beta_{1}+2 \beta_{2}+\cdots+2 \beta_{\ell-1}+\beta_{\ell} .
\end{gathered}
$$

The root space of the algebra $A_{2 \ell-1}^{(2)}(\ell \geq 3)$ may be constructed from the simple roots

$$
a_{0}=\left(-\beta_{1}-2 \beta_{2}-\cdots-2 \beta_{\ell-1}-\beta_{\ell}, 0, \frac{1}{2}\right), \quad a_{1}=\left(\beta_{1}, 0,0\right), \quad a_{\ell}=\left(\beta_{\ell}, 0,0\right) .
$$


The first element of the triplet for the root $a_{0}$ is the negative of the highest short root of $C_{\ell}$. The Cartan matrix for the $A_{2 \ell-1}^{(2)}$ is

$$
\left(\begin{array}{ccccccc}
2 & 0 & -1 & & & & \\
0 & 2 & -1 & & & 0 & \\
-1 & -1 & 2 & & & \\
& & & \ddots & & \\
& & & & 2 & -1 \\
& 0 & & & & \\
& & & & -2 & 2
\end{array}\right) .
$$

The null vector $(1,1,2, \ldots, 2,1)$ selects out the imaginary root

$$
\delta=\left(0,0, \frac{1}{2}\right)=a_{0}+a_{1}+2 a_{2}+\cdots+2 a_{\ell-1}+a_{\ell} .
$$

The positive imaginary roots for $A_{2 \ell-1}^{(2)}$ are

$$
\begin{gathered}
2 n \delta, \quad \text { where } n \in \mathbb{Z}_{+}-\{0\}, \\
n \delta, \quad \text { where } n \neq 0 \bmod 2 .
\end{gathered}
$$

The first set has a degeneracy $\ell$ and the second set has a degeneracy $(\ell-1)$. The real roots are nondegenerate. They are classified as:

$$
\begin{aligned}
& (\beta, 0,0) \cup( \pm \beta, 0, n), \quad \text { where } \beta \in \stackrel{\circ}{\Delta}_{L}, n \in \mathbb{Z}_{+}-\{0\}, \\
& (\beta, 0,0) \cup\left( \pm \beta, 0, \frac{n}{2}\right), \quad \text { where } \beta \in \stackrel{\circ}{\Delta}_{S}, n \in \mathbb{Z}_{+}-\{0\} .
\end{aligned}
$$

To discuss the contribution of the above sets of roots to the generating functional, we introduce the variable

$$
q=x_{0} x_{1}^{2} \cdots x_{\ell-1}^{2} x_{\ell},
$$

that is closely connected to the structure of $C_{\ell}$. Now the contribution of any particular series belonging to (4.12) may be understood by choosing $\beta=\beta_{\ell}$ (say), and thus obtaining the factor

$$
\left(1-x_{\ell}\right)^{-1} \prod_{n=1}^{\infty}\left[\left(1-q^{2 n} x_{\ell}\right)\left(1-q^{2 n} x_{\ell}^{-1}\right)\right]^{-1} .
$$

Neglecting the degeneracy factor, the imaginary roots in (4.10) contributes a factor

$$
\prod_{n=1}^{\infty}\left(1-q^{2 n}\right)^{-1} .
$$

The contributions (4.15) and (4.16) may be combined together as the inverse of the triple product factor

$$
\begin{aligned}
\prod_{n=1}^{\infty}( & {\left[1-\left(x_{0}^{2} x_{1}^{2} x_{2}^{4} \cdots x_{\ell-1}^{4} x_{\ell}\right)^{n} x_{\ell}^{n}\right]\left[1-\left(x_{0}^{2} x_{1}^{2} x_{2}^{4} \cdots x_{\ell-1}^{4} x_{\ell}\right)^{n-1} x_{\ell}^{n}\right] } \\
& \left.\times\left[1-\left(x_{0}^{2} x_{1}^{2} x_{2}^{4} \cdots x_{\ell-1}^{4} x_{\ell}\right)^{n} x_{\ell}^{n-1}\right]^{-1}\right)=\left[T\left(x_{\ell}, q^{2} x_{\ell}^{-1}\right)\right]^{-1} .
\end{aligned}
$$


Each of the series in (4.12) coupled with one of the $\boldsymbol{\ell}$-fold degenerate imaginary root series in (4.10) similarly generate an inverse of the triple product factor. Collecting together, we find this contribution to the generating functional for the KPF as

$$
\begin{aligned}
G_{L}\left(x_{1}, \ldots, x_{\ell}\right)=[ & T\left(x_{\ell}, q^{2} x_{\ell}^{-1}\right) T\left(x_{\ell-1}^{2} x_{\ell}, q^{2} x_{\ell-1}^{-2} x_{\ell}^{-2}\right) \\
& \left.\cdots T\left(x_{1}^{2} x_{2}^{2} \cdots x_{\ell-1}^{2} x_{\ell}, q^{2} x_{1}^{-2} x_{2}^{-2} \cdots x_{\ell-1}^{-2} x_{\ell}^{-1}\right)\right]^{-1} .
\end{aligned}
$$

The other series of the imaginary roots, listed in (4.11) has a multiplicity $(\ell-1)$ and it contributes to the generating functional

$$
\tilde{G}_{\operatorname{Im}}(q)=\prod_{n=1}^{\infty}\left(1-q^{2 n-1}\right)^{-(\ell-1)}=\left[\frac{\phi\left(q^{2}\right)}{\phi(q)}\right]^{\ell-1} .
$$

The real roots in (4.13) also make a contribution proportional to the inverse of the triple product structure. To illustrate, we consider the series in (4.13), where $\beta=\beta_{1}$. The contribution is

$$
\left(1-x_{1}\right)^{-1} \sum_{n=1}^{\infty}\left[\left(1-q^{n} x_{1}\right)\left(1-q^{n} x_{1}^{-1}\right)\right]^{-1}=\phi(q)\left[T\left(x_{1}, q x_{1}^{-1}\right)\right]^{-1} .
$$

The total contribution of all the real roots listed in (4.13) has the structure

$$
G_{S}\left(x_{1}, \ldots, x_{\ell}\right)=G_{A_{\ell-1}^{(1)}}\left(x_{1}, \ldots, x_{\ell-1} ; q\right) \tilde{G}_{S}\left(x_{1}, \ldots, x_{\ell}\right),
$$

where

$$
\begin{aligned}
& G_{A_{\ell-1}^{(1)}}\left(x_{1}, \ldots, x_{\ell-1} ; q\right) \\
&=[\phi(q)]^{(\ell-1)(\ell-2) / 2}\left[T\left(x_{1}, q x_{1}^{-1}\right) \cdots T\left(x_{\ell-1}, q x_{\ell-1}^{-1}\right) T\left(x_{1} x_{2}, q x_{1}^{-1} x_{2}^{-1}\right)\right. \\
&\left.\cdots T\left(x_{\ell-2} x_{\ell-1}, q x_{\ell-2}^{-1} x_{\ell-1}^{-1}\right) T\left(x_{1} x_{2} \cdots x_{\ell-1}, q x_{1}^{-1} \cdots x_{\ell-1}^{-1}\right)\right]^{-1},
\end{aligned}
$$

and

$$
\begin{aligned}
\tilde{G}_{S}\left(x_{1}, \ldots, x_{\ell}\right) & \\
=[\phi(q)]^{(\ell-1)(\ell+2) / 2}[ & T\left(x_{\ell-1} x_{\ell}, q x_{\ell-1}^{-1} x_{\ell}^{-1}\right) \cdots T\left(x_{1} \cdots x_{\ell}, q x_{1}^{-1} \cdots x_{\ell}^{-1}\right) \\
\times & T\left(x_{\ell-2} x_{\ell-1}^{2} x_{\ell}, q x_{\ell-2}^{-1} x_{\ell-1}^{-2} x_{\ell}^{-1}\right) \\
& \cdots T\left(x_{1} \cdots x_{\ell-2} x_{\ell-1}^{2} x, q x_{1}^{-1} \cdots x_{\ell-2}^{-1} x_{\ell-1}^{-2} x_{\ell}^{-1}\right) \\
& \left.\cdots T\left(x_{1} x_{2}^{2} \cdots x_{\ell-1}^{2} x_{\ell}, q x_{1}^{-1} x_{2}^{-1} x_{\ell-1}^{-2} x_{\ell}^{-1}\right)\right]^{-1} .
\end{aligned}
$$

The factor $G_{A_{\ell-1}^{(1)}}$ has the form of the generating functional for the KPF for the algebra $A_{\ell-1}^{(1)}$. The variables $q$ referred to in (4.21) and (3.17) are, however, different. Combining the factors (4.18), (4.19), and (4.21), we obtain the full generating functional for the KPF for an arbitrary algebra

$$
G_{A_{2 \ell-1}^{(2)}}=G_{L} \tilde{G}_{\mathrm{Im}} G_{S} .
$$

For the purpose of obtaining the recursive relation for the KPF, we restrict ourselves 
to the particular cases $A_{5}^{(2)}$. The technique is to substitute the generating functional $G_{A_{5}^{(2)}}$ as obtained from (4.24) to the left-hand side of (1.4) and then use the Euler identity (1.8) and triple product identity (1.9) for the appropriate arguments. Comparing terms with the same set of exponents of the variables $\left(x_{0}, x_{1}, \ldots, x_{\ell}\right)$ on both sides of (1.4), we get the recursive relation for the example of $A_{5}^{(2)}$ algebra

$$
\begin{gathered}
\sum_{\substack{m_{1}, m_{2}, m_{3}, n_{1}, \ldots, n_{6}, \in \mathbb{Z}}}(-1)^{\sum_{i=1}^{3} m_{i}+\sum_{j=1}^{6} n_{j}} K\left(N_{0}-\tilde{N}_{0}, N_{1}-\tilde{N}_{1}, N_{2}-\tilde{N}_{2}, N_{3}-\tilde{N}_{3}\right) \\
=\sum_{\substack{k_{1}, k_{2} \\
\ell_{1}, \ldots, \ell_{4} \\
\in \mathbb{Z}}}(-1)^{k_{1}+k_{2}+\ell_{1}+\cdots+\ell_{4}} \delta_{N_{0}, E} \delta_{N_{1}, E} \delta_{N_{2}, 2 E} \delta_{N_{3}, E},
\end{gathered}
$$

where

$$
\begin{aligned}
\tilde{N}_{0}= & \sum_{i=1}^{3} m_{i}\left(m_{i}-1\right)+\frac{1}{2} \sum_{j=1}^{6} n_{j}\left(n_{j}-1\right), \\
\tilde{N}_{1}= & \sum_{i=1}^{3} m_{i}\left(m_{i}-1\right)+\frac{n_{1}}{2}\left(n_{1}+1\right)+\frac{n_{2}}{2}\left(n_{2}-1\right)+\frac{n_{3}}{2}\left(n_{3}+1\right) \\
& +\frac{n_{4}}{2}\left(n_{4}-1\right)+\frac{n_{5}}{2}\left(n_{5}+1\right)-\frac{n_{6}}{2}\left(n_{6}-1\right), \\
\tilde{N}_{2}= & 2 \sum_{i=1}^{3} m_{i}^{2}-2 m_{1}+\sum_{i=1}^{6} n_{i}^{2}-n_{1}+n_{6}, \\
\tilde{N}_{3}= & \sum_{i=1}^{3} m_{i}^{2}+\frac{1}{2} \sum_{i=1}^{3} n_{i}\left(n_{i}-1\right)+\frac{1}{2} \sum_{i=4}^{6} n_{i}\left(n_{i}+1\right), \\
E= & \sum_{i=1,2} k_{i}\left(3 k_{i}+1\right)+\frac{1}{2} \sum_{i=1}^{4} \ell_{i}\left(3 \ell_{i}+1\right) .
\end{aligned}
$$

5. Partition functions for $D_{\ell+1}^{(2)}$. Now we construct the generating functional for the series $D_{\ell+1}^{(2)}$ (for $\ell \geq 2$ ). The Lie algebra $D_{\ell+1}$ has simple roots $\alpha_{1}, \alpha_{2}, \ldots, \alpha_{\ell+1}$ with the Dynkin diagram admitting the automorphism defined by the map

$$
\alpha_{\ell} \leftrightharpoons \alpha_{\ell+1},
$$

whereas the rest of the roots are kept invariant. The positive roots of $D_{\ell+1}$ that are invariant under the map (5.1) may be grouped in two categories:

(I) the roots of $A_{\ell-1}\left(\subset D_{\ell+1}\right)$ constructed from the simple roots

$$
\alpha_{1}, \alpha_{2}, \ldots, \alpha_{\ell-1}
$$

(II) the set consisting of

$$
\begin{aligned}
& \alpha_{\ell}+\alpha_{\ell-1}+\alpha_{\ell+1}, \alpha_{\ell-2}+\alpha_{\ell-1}+\alpha_{\ell}+\alpha_{\ell+1}, \\
& \ldots, \alpha_{1}+\cdots+\alpha_{\ell-1}+\alpha_{\ell}+\alpha_{\ell+1}, \alpha_{\ell-2}+2 \alpha_{\ell-1}+\alpha_{\ell}+\alpha_{\ell+1}, \\
& \ldots, \alpha_{1}+\cdots+\alpha_{\ell-2}+2 \alpha_{\ell-1}+\alpha_{\ell}+\alpha_{\ell+1}, \\
& \ldots, \alpha_{1}+2 \alpha_{2}+\cdots+2 \alpha_{\ell-1}+\alpha_{\ell}+\alpha_{\ell+1} .
\end{aligned}
$$


The subalgebra $B_{\ell}\left(\subset D_{\ell+1}\right)$ which is invariant under the map (5.1) is constructed from the simple roots where

$$
\begin{gathered}
\beta_{i}=\alpha_{i}, \quad i=1, \ldots,(\ell-1) ; \\
\beta_{\ell}=\frac{\alpha_{\ell}+\alpha_{\ell+1}}{2} .
\end{gathered}
$$

Previously we enlisted the roots of $B_{\ell}$. As evident from (3.5) and (3.6), the root of $D_{\ell+1}$ invariant under the automorphism (5.1) appear as the long roots of $B_{\ell}$. The simple roots of $D_{\ell+1}(\ell \geq 2)$ are listed below:

$$
a_{0}=\left(-\beta_{1}-\beta_{2}-\cdots-\beta_{\ell}, 0, \frac{1}{2}\right), \quad a_{1}=\left(\beta_{1}, 0,0\right), \quad a_{\ell}=\left(\beta_{\ell}, 0,0\right) .
$$

The first component of the root triple $a_{0}$ is the negative of the highest short root of $\beta_{\ell}$. The Cartan matrix for the algebra $D_{\ell+1}^{(2)}$ is

$$
\left(\begin{array}{ccccccc}
2 & -1 & 0 & & & \\
-2 & 2 & -1 & & & 0 & \\
0 & -1 & 2 & & & \\
& & & \ddots & & \\
& & & & 2 & -2 \\
& 0 & & & -1 & 2
\end{array}\right) \text {, }
$$

which admits a null vector $(1,1, \ldots, 1)$ that is related to the imaginary root

$$
\delta=\left(0,0, \frac{1}{2}\right)=a_{0}+a_{1}+\cdots+a_{\ell} .
$$

The complete series of the imaginary roots are:

$$
\begin{aligned}
& 2 n \delta, \quad \text { where } n \in \mathbb{Z}_{+}-\{0\}, \\
& n \delta, \quad \text { where } n \neq 0 \bmod 2 .
\end{aligned}
$$

The imaginary roots (5.8) have a degeneracy $\ell$ and the roots (5.9) are non-degenerate. The real roots of $D_{\ell+1}^{2}$ may also be grouped in two sets:

$$
(\beta, 0,0) \cup( \pm \beta, 0, n), \quad \text { where } \beta \in \stackrel{\circ}{\Delta}_{\ell} \text { as listed in (3.5) and (3.6); and } n \in \mathbb{Z}_{+}-\{0\} \text {, }
$$

$$
(\beta, 0,0) \cup\left( \pm \beta, 0, \frac{n}{2}\right), \quad \text { where } \beta \in \stackrel{\circ}{\Delta}_{s} \text { as listed in (3.4) and } n \in \mathbb{Z}_{+}-\{0\} .
$$

Following a procedure akin to the previously adopted one, we construct the generating functional for the KPF for an arbitrary algebra $D_{\ell+1}^{(2)}$. We introduce the variable

$$
q=x_{0} x_{1} \cdots x_{\ell}
$$

The generating functional may be conveniently grouped as a product of several factors. The series listed in (5.10) with $\beta$ taken as root of the subalgebra $A_{\ell-1}\left(\subset B_{\ell}\right)$, as listed in (3.5) produces a factor $G_{A_{\ell-1}}^{(1)}\left(x_{1}, \ldots, x_{\ell-1}, q^{2}\right)$ characteristic of the generating KPF functional for $A_{\ell-1}^{(1)}$ algebra. This factor which also includes part of the 
contribution from the imaginary roots is

$$
\begin{aligned}
G_{A_{\ell-1}^{(1)}}\left(x_{1}, \ldots, x_{\ell-1} ; q^{2}\right) & \\
=\left[\phi\left(q^{2}\right)\right]^{(\ell-1)(\ell-2) / 2} & {\left[T\left(x_{1}, q^{2} x_{1}^{-1}\right) \cdots T\left(x_{\ell-1}, q^{2} x_{\ell-1}^{-1}\right)\right.} \\
& \times T\left(x_{1} x_{2}, q^{2} x_{1}^{-1} x_{2}^{-1}\right) \cdots T\left(x_{\ell-2} x_{\ell-1} ; q^{2} x_{\ell-2}^{-1} x_{\ell-1}^{-1}\right) \\
& \left.\cdots T\left(x_{1} x_{2} \ldots, x_{\ell-1}, q^{2} x_{1}^{-1} x_{2}^{-1} \cdots x_{\ell-1}^{-1}\right)\right] .
\end{aligned}
$$

The rest of the contribution from the imaginary roots is $[\phi(q)]^{-1}$. The contribution corresponding to the other long roots of $B_{\ell}$ listed in (3.6) may be grouped as

$$
\begin{aligned}
& \tilde{G}_{L}\left(x_{1}, \ldots, x_{\ell}\right) \\
& =\left[\phi\left(q^{2}\right)\right]^{\ell(\ell-1) / 2}\left[T\left(x_{\ell-1} x_{\ell}^{2}, q^{2} x_{\ell-1}^{-1} x_{\ell}^{-2}\right) \cdots T\left(x_{1} \cdots x_{\ell-1} x_{\ell}^{2}, q^{2} x_{1}^{-1} \cdots x_{\ell-1}^{-1} x_{\ell}^{-2}\right)\right. \\
& \cdots T\left(x_{\ell-2} x_{\ell-1}^{2} x_{\ell}^{2}, q^{2} x_{\ell-2}^{-1} x_{\ell-1}^{-2} x_{\ell}^{-2}\right) \\
& \left.\cdots T\left(x_{1} x_{2}^{2} \cdots x_{\ell}^{2}, q^{2} x_{1}^{-1} x_{2}^{-2} \cdots x_{\ell}^{-2}\right)\right]^{-1} \text {. }
\end{aligned}
$$

The contribution corresponding to the short roots of $B_{\ell}$ listed in (3.4) is

$$
\begin{array}{r}
G_{S}\left(x_{1}, \ldots, x_{\ell}\right)=[\phi(q)]^{6}[ \\
T\left(x_{\ell}, q x_{\ell}^{-1}\right) T\left(x_{\ell-1} x_{\ell}, q x_{\ell-1}^{-1} x_{\ell}^{-1}\right) \\
\left.\cdots T\left(x_{1} \cdots x_{\ell}, q x_{1}^{-1} \cdots x_{\ell}^{-1}\right)\right]^{-1} .
\end{array}
$$

Combining the different contributions, the full generating functional emerges as

$$
G_{D_{\ell+1}}^{(2)}\left(x_{1}, \ldots, x_{\ell}\right)=[\phi(q)]^{-1} G_{A_{\ell-1}^{(1)}}\left(x_{1}, \ldots, x_{\ell-1}\right) \cdot \tilde{G}_{L}\left(x_{1}, \ldots, x_{\ell}\right) G_{S}\left(x_{1}, \ldots, x_{\ell}\right) \text {. }
$$

The explicit construction of the generating functional in terms of the inverse of the triple product and the Euler product may be used to derive the recursive relations as done previously. We just quote the formula for the $D_{4}^{(2)}$ algebra:

$$
\begin{aligned}
\sum_{\substack{m_{1}, \ldots, m_{6} \\
n_{1}, n_{3}, n_{3}}}(-1)^{\sum_{i=1}^{6} m_{i}+\sum_{j=1}^{3} n_{j}} K\left(N_{0}-\tilde{N}_{0}, N_{1}-\tilde{N}_{1}, N_{2}-\tilde{N}_{2}, N_{3}-\tilde{N}_{3}\right) \\
=\sum_{\substack{k_{1}, k_{2}, k_{3}, k_{4} \\
\ell_{1}, \ell_{2} \in \mathbb{Z}}}(-1)^{\sum_{i=1}^{4} k_{i}+\sum_{j=1}^{2} \ell_{j}} \delta_{N_{1}, E} \delta_{N_{2}, E} \delta_{N_{3}, E} \delta_{N_{0}, E},
\end{aligned}
$$

where

$$
\begin{aligned}
& \tilde{N}_{0}=\sum_{i=1}^{6} m_{i}\left(m_{i}-1\right)+\frac{1}{2} \sum_{i=1}^{3} n_{i}\left(n_{i}-1\right) \\
& \tilde{N}_{1}=\sum_{i=1}^{6} m_{i}^{2}-m_{2}-m_{4}+\frac{n_{1}}{2}\left(n_{1}-1\right)+\frac{n_{2}}{2}\left(n_{2}-1\right)+\frac{n_{3}}{2}\left(n_{3}+1\right), \\
& \tilde{N}_{2}=\sum_{i=1}^{6} m_{i}^{2}-m_{1}+m_{6}+\frac{n_{1}}{2}\left(n_{1}-1\right)+\frac{n_{2}}{2}\left(n_{2}+1\right)+\frac{n_{3}}{2}\left(n_{3}-1\right), \\
& \tilde{N}_{3}=\sum_{i=1}^{6} m_{i}\left(m_{i}-1\right)+\sum_{i=4}^{6} m_{i}\left(m_{i}+1\right)+\frac{1}{2} \sum_{i=1}^{3} n_{i}\left(n_{i}+1\right), \\
& E=\sum_{i=1}^{4} k_{i}\left(3 k_{i}+1\right)+\frac{1}{2} \sum_{i=1}^{2} \ell_{i}\left(3 \ell_{i}+1\right) .
\end{aligned}
$$


6. Partition functions for $E_{6}^{(2)}$ and $D_{4}^{(3)}$. The simple roots $\alpha_{1}, \ldots, \alpha_{6}$ of the Lie algebra $E_{6}$ admit the automorphism of its Dynkin diagram given by the map

$$
\alpha_{1} \leftrightharpoons \alpha_{6}, \quad \alpha_{2} \leftrightharpoons \alpha_{5},
$$

and the other roots remain unaltered. The simple roots of the invariant subalgebra $F_{4}$ are projected as

$$
\beta_{1}=\frac{\alpha_{1}+\alpha_{6}}{2}, \quad \beta_{2}=\frac{\alpha_{2}+\alpha_{5}}{2}, \quad \beta_{3}=\alpha_{3}, \quad \beta_{4}=\alpha_{4}
$$

The invariant roots of $E_{6}$ map to the long roots of $F_{4}$. The simple roots of the algebra $E_{6}^{(2)}$ may be chosen as

$$
a_{0}=\left(-2 \beta_{1}-3 \beta_{2}-2 \beta_{3}-\beta_{4}, 0, \frac{1}{2}\right), \quad a_{i}=\left(\beta_{i}, 0,0\right),
$$

where $i=1,2,3,4$. The first component of the triplet for the root $a_{0}$ is just the negative of the highest short root of $F_{4}$. We just quote the result for the generating functional $G_{E_{6}^{(2)}}\left(x_{0}, x_{1}, \ldots, x_{4}\right)$ having the multiplicative structure

$$
G_{E_{6}^{(2)}}=G_{L} G_{\operatorname{Im}} G_{S}
$$

where $G_{L}$ refers to the contribution due to those root sequences of $E_{6}^{(2)}$ which relate to the invariant roots of the Lie algebra $E_{6}$, and consequently to the long roots of $F_{4}$. The factor $G_{S}$ indicates the contribution of the root series of $E_{6}^{(2)}$ corresponding to the short roots of $F_{4}$. The imaginary roots generate the term $F_{4}$. The explicit values of these factors are given below:

$$
\begin{aligned}
G_{L}\left(x_{0}, x_{1}, x_{2}, x_{3}, x_{4}\right)=[ & \left.\phi\left(q^{2}\right)\right]^{12} \\
{[} & T\left(x_{3}, q^{2} x_{3}^{-1}\right) T\left(x_{4}, q x_{4}^{-1}\right) T\left(x_{3} x_{4}, q x_{3}^{-1} x_{4}^{-1}\right) \\
& \times T\left(x_{2}^{2} x_{3}, q^{2} x_{2}^{-2} x_{3}^{-1}\right) T\left(x_{2}^{2} x_{3} x_{4}, q^{2} x_{2}^{-2} x_{3}^{-1} x_{4}^{-1}\right) \\
& \times T\left(x_{1}^{2} x_{2}^{2} x_{3}, q^{2} x_{1}^{-2} x_{2}^{-2} x_{3}^{-1}\right) T\left(x_{2}^{2} x_{3}^{2} x_{4}, q^{2} x_{2}^{-2} x_{3}^{-2} x_{4}^{-1}\right) \\
& \times T\left(x_{1}^{2} x_{2}^{2} x_{3} x_{4}, q^{2} x_{1}^{-2} x_{2}^{-2} x_{3}^{-1} x_{4}^{-1}\right) \\
& \times T\left(x_{1}^{2} x_{2}^{2} x_{3}^{2} x_{4}, q^{2} x_{1}^{-2} x_{2}^{-2} x_{3}^{-2} x_{4}^{-1}\right) \\
& \times T\left(x_{1}^{2} x_{2}^{4} x_{3}^{2} x_{4}, q^{2} x_{1}^{-2} x_{2}^{-4} x_{3}^{-2} x_{4}^{-2}\right) \\
& \times T\left(x_{1}^{2} x_{2}^{4} x_{3}^{3} x_{4}, q^{2} x_{1}^{-2} x_{2}^{-4} x_{3}^{-3} x_{4}^{-1}\right) \\
& \left.\times T\left(x_{1}^{2} x_{2}^{4} x_{3}^{3} x_{4}^{2}, q^{2} x_{1}^{-2} x_{2}^{-4} x_{3}^{-3} x_{4}^{-2}\right)\right], \\
& G_{\operatorname{Im}}(q)=\left[\phi\left(q^{2}\right) \phi(q)\right]^{-2},
\end{aligned}
$$




$$
\begin{aligned}
G_{S}\left(x_{0}, x_{1}, x_{2}, x_{3}, x_{4}\right)=[ & \phi(q)]^{12} \\
{[} & T\left(x_{1} q x_{1}^{-1}\right) T\left(x_{2}, q x_{2}^{-1}\right) \\
& \times T\left(x_{1} x_{2}, q x_{1}^{-1} x_{2}^{-1}\right) T\left(x_{2} x_{3}, q x_{2}^{-1} x_{3}^{-1}\right) \\
& \times T\left(x_{1} x_{2} x_{3}, q x_{1}^{-1} x_{2}^{-1} x_{3}^{-1}\right) T\left(x_{2} x_{3} x_{4}, q x_{2}^{-1} x_{3}^{-1} x_{4}^{-1}\right) \\
& \times T\left(x_{1} x_{2}^{2} x_{3}, q x_{1}^{-1} x_{2}^{-2} x_{3}^{-1}\right) T\left(x_{1} x_{2} x_{3} x_{4}, q x_{1}^{-1} x_{2}^{-1} x_{3}^{-1} x_{4}^{-1}\right) \\
& \times T\left(x_{1} x_{2}^{2} x_{3} x_{4}, q x_{1}^{-1} x_{2}^{-2} x_{3}^{-1} x_{4}^{-1}\right) \\
& \times T\left(x_{1} x_{2}^{2} x_{3}^{2} x_{4}, q x_{1}^{-1} x_{2}^{-2} x_{3}^{-2} x_{4}^{-1}\right) \\
& \times T\left(x_{1} x_{2}^{3} x_{3}^{2} x_{4}, q x_{1}^{-1} x_{2}^{-3} x_{3}^{-2} x_{4}^{-1}\right) \\
& \left.\times T\left(x_{1}^{2} x_{2}^{3} x_{3}^{2} x_{4}, q x_{1}^{-2} x_{2}^{-3} x_{3}^{-2} x_{4}^{-1}\right)\right]
\end{aligned}
$$

where

$$
q=x_{0} x_{1}^{2} x_{2}^{3} x_{3}^{2} x_{4}
$$

Derivation of the recursive relation for the KPF is straightforward and we omit it here.

The example of the algebra $D_{4}^{(3)}$ is distinct from the others because the Dynkin diagram of the Lie algebra $D_{4}$ is symmetric under the permutation group $S_{3}$ for the simple roots $\alpha_{1}, \alpha_{2}$, and $\alpha_{3}$ thus defining an automorphism of order 3:

$$
\tau^{3}=1
$$

The subalgebra of $D_{4}$, invariant under the automorphism may be projected as

$$
\beta_{1}=\frac{\alpha_{1}+\alpha_{2}+\alpha_{3}}{3}, \quad \beta_{2}=\beta_{4}
$$

The roots of $D_{4}$, invariant under $\tau$ map to the long roots of $G_{2}$. We enlist the simple roots of $D_{4}^{(3)}$ as

$$
a_{0}=\left(-2 \beta_{1}-\beta_{2}, 0, \frac{1}{3}\right), \quad a_{i}=\left(\beta_{i}, 0,0\right),
$$

where $i=1,2$. The first component of the root is negative of the highest short root of $G_{2}$. The Cartan matrix for $D_{4}^{(3)}$ is

$$
\left(\begin{array}{ccc}
2 & -1 & 0 \\
-1 & 2 & -1 \\
0 & -3 & 2
\end{array}\right)
$$

which admits a null vector $(1,2,1)$ relating to the imaginary root

$$
\delta=\left(0,0, \frac{1}{3}\right)=a_{0}+2 a_{1}+a_{2} .
$$

The imaginary roots appear in two series:

(I) the roots $3 n \delta$, where $n \in \mathbb{Z}_{+}-\{0\}$ and the multiplicity is 2 , and

(II) the non-degenerate root $n \delta$, where $n \neq 0 \bmod 3$. 
The real roots of $D_{4}^{(3)}$ are of two kinds. The roots of the first type owe their origin to the invariant roots of the Lie algebra $D_{4}$. They are

$$
(\beta, 0,0) \cup( \pm \beta, 0, n),
$$

where $n \in \mathbb{Z}_{+}-0$ and $\beta$ is a positive long root of $G_{2}$. The second type may be listed as

$$
(\beta, 0,0) \cup\left( \pm \beta, 0, \frac{n}{3}\right),
$$

where $n \in \mathbb{Z}_{+}-0$ and $\beta$ is a positive short root of $G_{2}$. To write the generating functional, we introduce

$$
q=x_{0} x_{1}^{2} x_{2}
$$

The contribution of the imaginary roots to the generating functional is

$$
G_{\operatorname{Im}}(q)=\left[\phi\left(q^{3}\right)\right]^{-2} \sum_{n=1}^{\infty}\left[\left(1-q^{3 n-2}\right)\left(1-q^{3 n-1}\right)\right]^{-1}=\left[\phi\left(q^{3}\right) \phi(q)\right]^{-1} .
$$

The real roots of the series (6.14) contribute

$G_{L}\left(x_{0}, x_{1}, x_{2}, x_{3}\right)=\left[\phi\left(q^{3}\right)\right]^{3}\left[T\left(x_{2}, q^{2} x_{2}^{-1}\right) T\left(x_{1}^{3} x_{2}, q^{2} x_{1}^{-3} x_{2}^{-1}\right) T\left(x_{1}^{3} x_{2}^{2}, q^{2} x_{1}^{-3} x_{2}^{-2}\right)\right]^{-1}$.

The other real roots appearing in (6.15) contribute

$$
G_{S}\left(x_{0}, x_{1}, x_{2}, x_{3}\right)=[\phi(q)]^{3}\left[T\left(x_{1}, q x_{1}^{-1}\right) T\left(x_{1} x_{2}, q x_{1}^{-1} x_{2}^{-1}\right) T\left(x_{1}^{2} x_{2}, q x_{1}^{-2} x_{2}^{-1}\right)\right]^{-1} \text {. }
$$

The full generating functional for the KPF has the structure

$$
G=G_{\operatorname{Im}} G_{L} G_{S} .
$$

The recursive relation for the KPF may be derived as before and reads

$$
\begin{array}{r}
\sum_{\substack{m_{1}, m_{2}, m_{3} \\
n_{1}, n_{2}, m_{3} \\
\in \mathbb{Z}}}(-1)^{\sum_{i=1}^{3} m_{i}+n_{i}} K\left(N_{0}-\tilde{N}_{0}, N_{1}-\tilde{N}_{1}, N_{2}-\tilde{N}_{2}\right) \\
=\sum_{k_{1}, k_{2}, \ell_{1}, \ell_{2}}(-1)^{k_{1}+k_{2}+\ell_{1}+\ell_{2}} \delta_{N_{0}, E} \delta_{N_{1}, 2 E} \delta_{N_{2}, E},
\end{array}
$$

where

$$
\begin{aligned}
\tilde{N}_{0} & =\frac{3}{2} \sum_{i=1}^{3} m_{i}\left(m_{i}-1\right)+\frac{1}{2} \sum_{i=1}^{3} n_{i}\left(n_{i}-1\right), \\
\tilde{N}_{1} & =3 \sum_{i=1}^{3} m_{i}^{2}-3 m_{1}+\sum_{i=1}^{3} n_{i}^{2}+n_{3}, \\
\tilde{N}_{2} & =\frac{3}{2} \sum_{i=1}^{3} m_{i}^{2}-\frac{1}{2} \frac{m_{1}+m_{2}-m_{3}}{2}+\frac{1}{2} \sum_{i=1}^{3} n_{i}^{2}-\frac{n_{1}-n_{2}+n_{3}}{2}, \\
E & =\frac{3}{2} \sum_{i=1}^{2} k_{i}\left(3 k_{i}+1\right)+\frac{1}{2} \sum_{i=1}^{2} \ell_{i}\left(3 \ell_{i}+1\right) .
\end{aligned}
$$


7. Conclusion. We obtained the general structure of the generating functional for the KPF for an arbitrary twisted affine algebra. The recursive relation for the KPF may be obtained in any specific example by a simple algorithm. It is noteworthy that for all of the affine algebras, both twisted and untwisted ones, we need to use only the identities (1.8), (1.9), and (1.10) to extract these recursion relations. The series $A_{2 \ell}^{(2)}$ is further distinguished in the sense that only for them the quintuple product structure appear in the generating functional thus necessitating use of the identity (1.10). The reason can be traced back to the fact that the longest roots among the roots of these different sizes always contain the smallest simple root in a multiple of 4 , thus securing the appropriate structure for the inverse of the quintuple product structure in the generating functional.

A characteristic infinite product identity for each affine Lie algebra may be derived by exploiting the generating functional approach and the alternate formulation of (Kac and Peterson [4]). The relations of these identities with the modular functions are under investigation and will be the subject of a future publication.

\section{REFERENCES}

[1] L. Carlitz, Generating Functions and Partition Problems, Proc. Sympos. Pure Math., Vol. VIII, Amer. Math. Soc., Providence, R.I., 1965, pp. 144-169. MR 31\#72. Zbl 142.25104.

[2] P. Goddard and D. Olive (eds.), Kac-Moody and Virasoro Algebras, World Scientific Publishing Co., Singapore, 1988. MR 89f:17022. Zbl 661.17001.

[3] V. G. Kac, Infinite-dimensional Lie algebras, and the Dedekind $\eta$-function, Funkcional. Anal. i Priložen. 8 (1974), no. 1, 77-78. MR 51\#10410. Zbl 299.17005.

[4] V. G. Kac and D. H. Peterson, Infinite-dimensional Lie algebras, theta functions and modular forms, Adv. in Math. 53 (1984), no. 2, 125-264. MR 86a:17007. Zbl 584.17007.

[5] T. S. Santhanam and R. Chakrabarti, Kostant partition functions for affine Kac-Moody algebras, Internat. J. Math. Math. Sci. 20 (1997), no. 3, 539-552. MR 98e:17033. Zbl 881.17023.

[6] J. Tannery and J. Molk, Éléments de la Théorie des Fonctions Elliptiques. Tome III: Calcul Intégral. Première Partie. Tome IV: Calcul Intégral. Deuxième Partie, Chelsea Publishing Co., Bronx, N.Y., 1972. MR 52\#13289. Zbl 296.33003.

Ranabir Chakrabarti: DePartment of Theoretical Physics, University of Madras, MADRAS 600025, INDIA

Thalanayar S. SANThanam: DePartment of Physics, PARKs College of ENGineERING AND AVIATION, SAINT LOUIS UNIVERSITY, SAINT LOUis, MisSouri 63103, USA

E-mail address: santhap3@slu. edu 


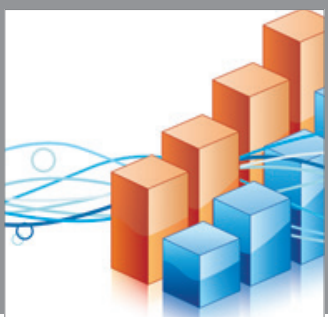

Advances in

Operations Research

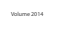

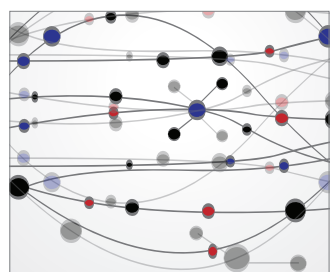

\section{The Scientific} World Journal
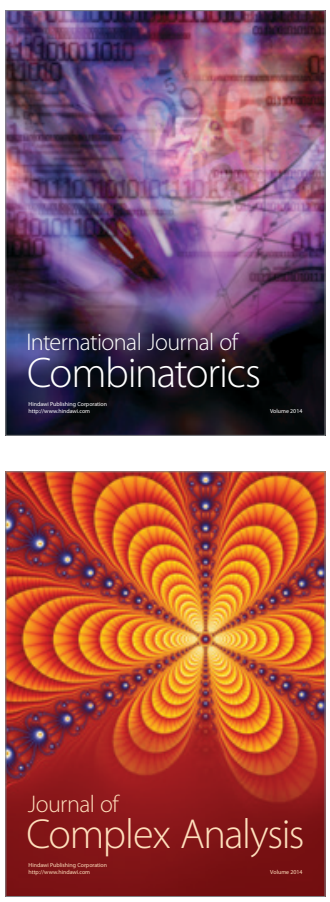

International Journal of

Mathematics and

Mathematical

Sciences
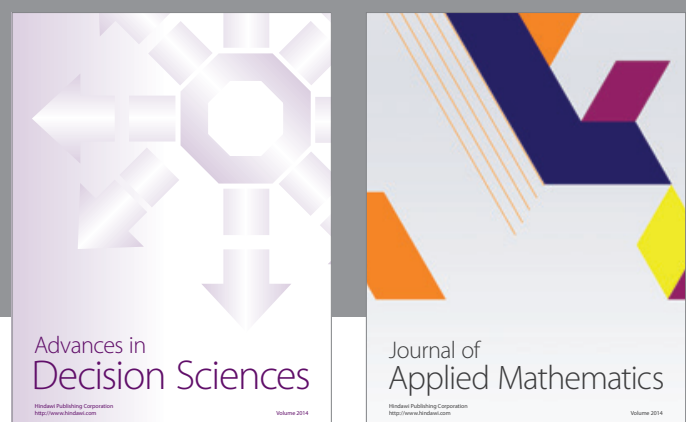

Journal of

Applied Mathematics
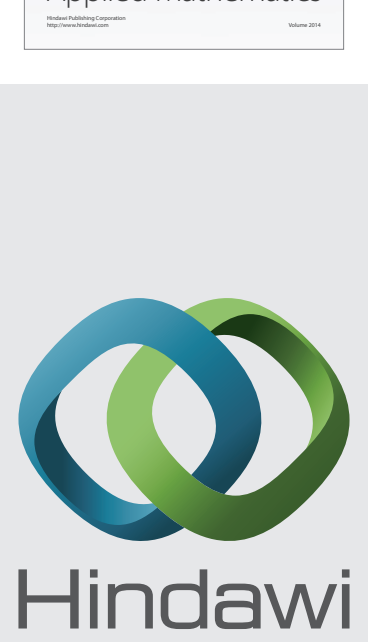

Submit your manuscripts at http://www.hindawi.com
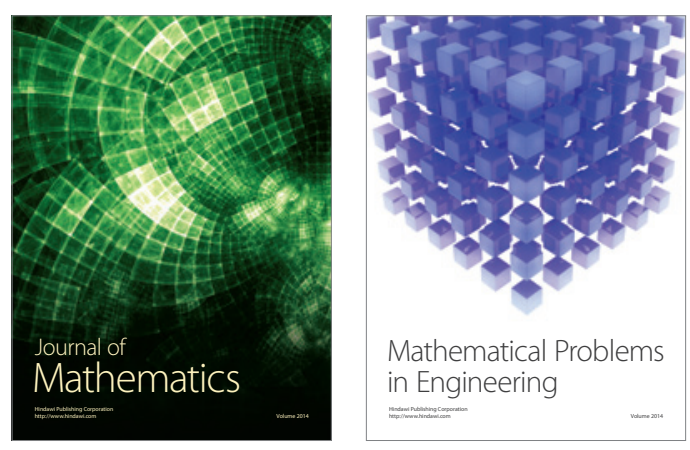

Mathematical Problems in Engineering
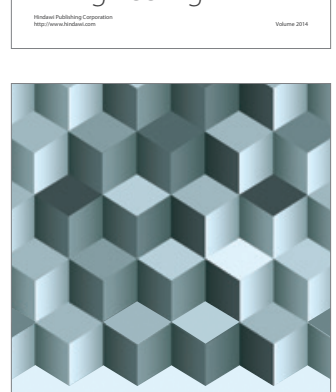

Journal of

Function Spaces
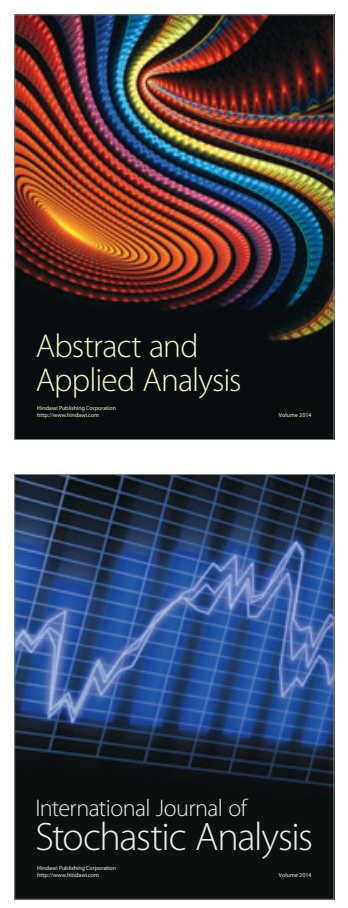

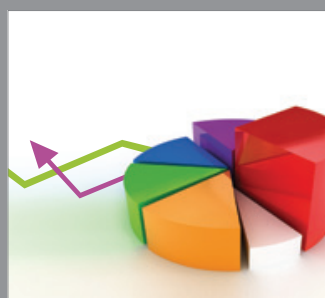

ournal of

Probability and Statistics

Promensencen
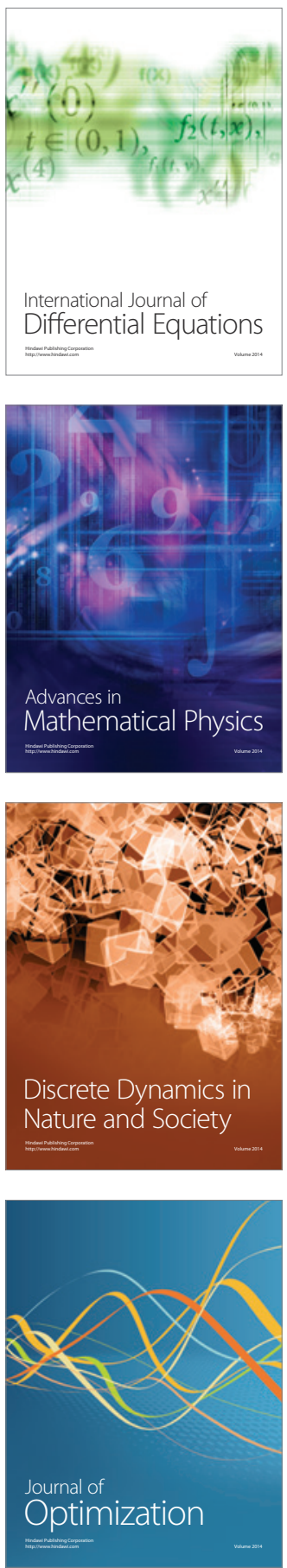